\title{
Adaptive Subband Selection in OFDM-BasedCognitive Radios
}
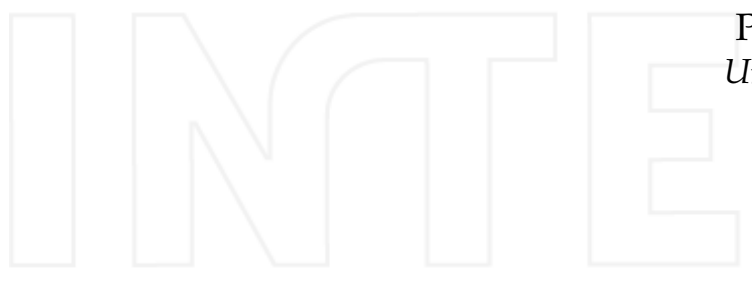

Pingzhou Tu, Xiaojing Huang University of Wollongong, Australia

Eryk Dutkiewicz

Macquarie University, Australia

\section{Introduction}

The cognitive radio built on software-defined radio enables intelligent wireless communication systems (S. Haykin, 2005). Orthogonal frequency division multiplexing (OFDM) as an promising cognitive radio modulation technique provides great flexibility and adaptability in selecting system transmission bandwidth for interference avoidance among coexistent multiple wireless systems in a shared radio environment (G. Andrea, 2006).

An important issue in achieving system coexistence is to deal with the interference generated by multiple wireless systems. When they are deployed in the proximity of each other, share the same frequency band, and operate at the same time, they are more likely to interfere with each other.

Many parameters affect the inteference between different wireless systems, such as power levels, modulation types, amount of data traffic, and separation between antenanas. Medium access control (MAC) layer protocol can also play a very important role in the interfernece avoidance and affect the overal system performance.

Some other multiple access methods such as TDMA, FDMA, and CDMA are effective in solving the coexitence problem among multiple wireless devices in the same networks respectively, but how different wireless systems can coexist in the same frequency band is only considered resently. The first standards-making body to consider the multiple wireless system coexistence problem is the IEEE 802.15 Working Group. IEEE 802.15.2 was formed to develop a recommended practice for coexistence between Bluetooth/IEEE 802.15.1 and IEEE 802.11, in which two types of coexistence mechanisms, collaborative and noncollaborative ones, are developed. With collaborative mechanism, wireless personal area networks and wireless local area networks exchange information over a separate link between one another to minimize mutual interference. In noncollaborative systems, there are several methods used for interference suppression. However, these methods are only implementations of the distributed coordination function (DCF) channel access mechanism for IEEE 802.11 to suppress interference (T. Cooklev, 2004). 
In this chapter, a novel adaptive subband selection method combined with an interleaved spread sepctrum OFDM (ISS-OFDM) technique is proposed to improve system coexistence in a shared spectrum environment such as the industrial, scientific, and medical

(ISM) band. It uses noncollorative mechanism and is a physical layer technique for system coexistence. The system performance such as the transmitted signal peak to average power ratios (PAR) (S. B. Slimane, 2002), and the system bit error rates (BER) (J. G. Proakis, 2000) before and after subband selection in different channel conditions are analyzed and simulated. The results show that the ISS-OFDM technique with adaptive subband selection can significantly improve the system performance when multiple systems coexist in a shared radio channel.

The contributions of this chapter are three-fold:

a. A novel complex exponential spreading (CES) method is developed to generate a spreading spectrum OFDM signal with multiple subbands.

b. The demodulation by using multiple fast Fourier transform (FFT) parallel operations is proposed to recover the transmitted information, which greatly reduces the receiver computational complexity and makes full use of signal diversity.

c. An adaptive subband selection transmission algorithm is proposed. Based on dynamically determined interference thresholds, system transmission band is adaptively selected to avoid interference among OFDM systems and other narrowband or ultra-wideband wireless systems.

This chapter consists of the following sections. Section 2 describes the proposed cognitive radio system model, and presents the ISS-OFDM technique by which the cognitive radio transmitter can generate signal with multiple subbands. The signal subbands used for system transmission can be decided based on channel sensing. Section 3 proposes the adaptive subband selection method and derives the interference power thresholds. Based on these thresholds, the cognitive radio receiver can decide if a subband should be selected or discarded in order to achieve the best system performance. The rule is that any subband with interference power level above an adaptively determined threshold will be discarded and the signals from weak or zero interference subbands will be decoded and combined to improve the signal-to-noise ratio. Section 4 provides simulation results on system performance, such as PAR and BER before and after subband selection in different channel conditions. Finally, Section 5 draws conclusions.

\section{Cognitive Radio System Model}

In this section, we describe the proposed baseband system model of the OFDM-based cognitive radios as displayed in Figure 1. Then, the generation and reception techniques of the ISS-OFDM signals are discussed. We assume that the data bit to symbol mapping scheme on the ISS-OFDM baseband system model is QPSK, the channel is frequency selective with slow fading, and the noise is AWGN with zero mean.

\subsection{Transmitter Architecture}

The function of the transmitter is to generate an ISS-OFDM signal with time and frequency diversities. At the transmitter, the complex QPSK data symbols to be transmitted are first divided into an $N$ by 1 vector, where $N$ is the number of subcarriers. This vector modulate $N$ subcarries in parallel by using a spread spectrum modulation technique, called complex 
exponential spreading (CES). Each data symbol modulates its corresponding subcarrier multiple times, and several different samples bearing the same data information can be generated. The replicas of the same data information are interleaved and then transmitted in different time slots, instead of adding the modulated samples together. After the spread spectrum modulation, an ISS-OFDM symbol is generated.

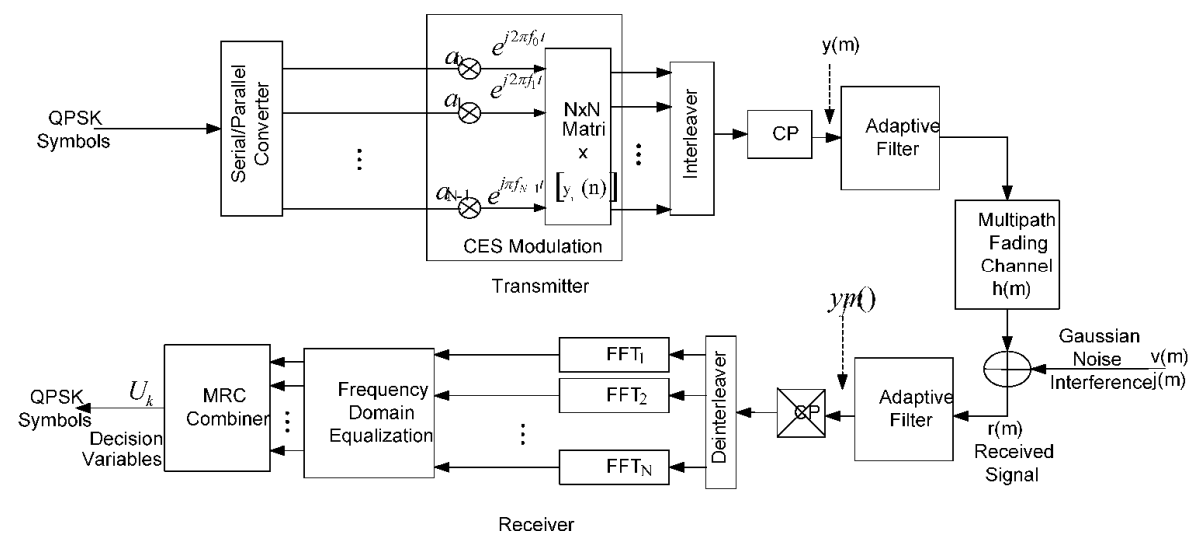

Fig. 1. Baseband system model.

\subsubsection{Complex Exponential Spreading (CES) Modulator}

For convenience, we will only describe the modulation process for one OFDM symbol. After serial to parallel conversion, each of the QPSK symbols modulates the corresponding subcarrier of the $N$ subcarriers. If the ISS-OFDM symbol period is $T_{s}, f_{i}=i / T_{s}$ denotes the $i^{\text {th }}$ subcarrier frequency of the $N$ orthogonal subcarriers, and $a_{i}$ modulates the $i^{\text {th }}$ subcarrier at time $t=\frac{n}{N} T_{S}, n=0,1, \ldots, N 1$, where $n$ is the sampling interval, the modulated symbol on the $i^{\text {th }}$ subcarrier and the $n^{\text {th }}$ time index is written as:

$$
y_{i}(n)=a_{i} e^{j 2 \pi f_{i} t}=a_{i} e^{j 2 \pi n i / N} .
$$

In ISS-OFDM symbol duration $T_{s}$, each element of the $N \times 1$ data symbol vector modulates the same corresponding subcarrier $N$ times. $N$ elements in the vector generate $N \times N$ matrix samples after modulation.

\subsubsection{Interleaving}

After the modulation, the $N_{x}$ Nmatrix samples are interleaved. Interleaving is used to obtain time diversity in a digital communications system without adding much overhead. The function of the interleaver is to spread the OFDM modulated signal samples out in time so that if there is deep fading or a noise burst, the signal samples from the modulated signal are not corrupted at the same time. 
There are many forms of interleaving techniques to implement the permutation of the signal samples in the matrix of the CES modulator output. A periodical interleaver is the simplest method to permute the transmission sequence. For the simplicity of description, we adopt the periodic interleaving algorithm. For example, for the I $x \mathrm{~N}$ matrix for one OFDM symbol, the interleaver writes the modulated samples for each of the same subcarrier row by row to form the I $x \mathrm{~N}$ matrix and reads the samples from the matrix column by column and places the samples on a time axis, which is displayed in Figure 2.

Mathematically, if the $I x N$ matrix is denoted as $\left[y_{i}(\mathrm{n})\right], i=0,1,-, I-1, n=0,1,-, N-1$, and $I, N$ are integers. Let the period of the interleaving be $N$, after periodically interleaving, the interleaved serial sequence can be written as follows:

$$
y(m)=y(n N+i)=y_{i}(n)=a_{i} e^{j 2 \pi f_{i} t}=a_{i} e^{j 2 \pi n i / N}
$$

where $i=0,1,-, I-1, n=0,1,-, N-1$.

\subsubsection{Generation of Multiple Subband OFDM Signal}

In the following, we discuss interleaved results by using the periodic interleaving alogrithm. We implement the periodic interleaving by shifting $N$ modulated subcarriers on a different time slots and adding them together. For instance, the $i^{\text {th }}$ subcarrier of the $N$ subcarriers is shifted by $i \cdot{ }_{T}$, where $T$ is the sampling interval, $T=N$. Then, the $N$ shifted subcarriers are added together to form one ISS-OFDM symbol with $N^{2}$ samples. The $m^{\text {th }}$ sample in the ISS-OFDM symbol, denoted by $\mathrm{y}(\mathrm{m}), \mathrm{m}=n N+i=0,1,-, \mathrm{N}^{2}-1$. , can be mathematically written as follows:

$$
y(m)=\sum_{i=1}^{N} \sum_{n}^{N} y_{i}(n) \delta[\mathrm{m}-\mathrm{i}-\mathrm{n} N],
$$

where $\delta[m-n N-i]=\left\{\begin{array}{l}1, \text { for } m=n N+i \\ 0, \text { for } m \neq n N+i\end{array}\right.$ is the unit impulse. If periodical interleaving is performed, taking out $\mathrm{y}(\mathrm{m})$ for each $m=n N+i, y(\mathrm{~m})$ can be simplified in the form: $y(m)=y(n N+i)=y_{i}(n), m=n N+i$, and $n, i=0,1, \ldots, N-1$. Thus, $N^{2}$ samples are produced in the same symbol period $T$, and the number of samples is spread $N$ times. The sampling rate is $N^{2} / T$. an ISS-OFDM symbol is generated. Figure 3 shows the signal modulation and periodical interleaving process for generating an ISS-OFDM symbol in the case of subcarriers number $N=4$. It is seen that the modulated samples on the $i^{\text {th }}(i=0,1,2,3)$ subcarrier are shifted $i$ time intervals, and then they are added on the time axis, and the number of samples increases to $N^{2}$ from the original number $N$. In the frequency domain the ISS-OFDM signal can be written as:

$$
\begin{aligned}
y(k) & =F F T(y(m))=F F T(y(n N+i)) \\
& =\sum_{n N+i=0}^{N^{2}-1} y(n N+i) e^{-j \frac{2 \pi}{N^{2}}(n N+i) k} .
\end{aligned}
$$




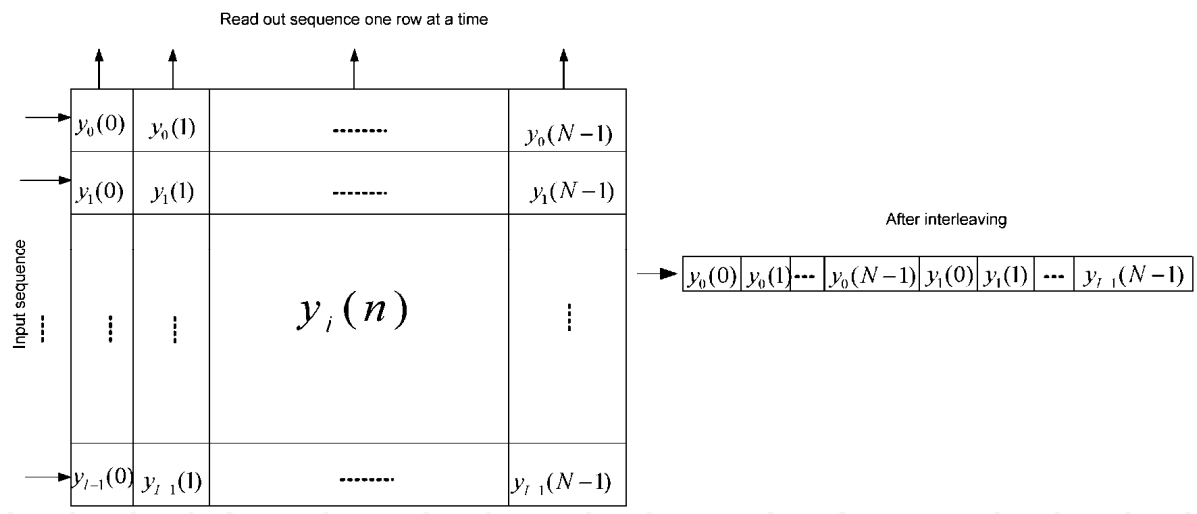

Fig. 2. Periodical interleaving.
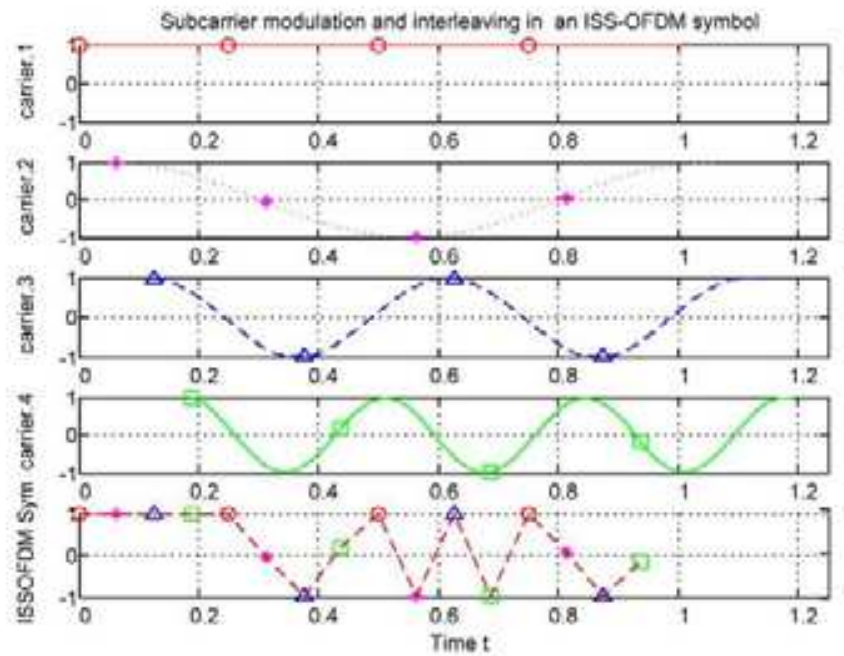

Fig. 3. Modulation and periodical interleaving process with subcarrier number $N=4$.

That is,

$$
y(k)=\sum_{n=0}^{N-1} \sum_{i=0}^{N-1} y(n N+i) e^{-j \frac{2 \pi}{N^{2}}(n N+i) k}
$$

Substituting $y(\mathrm{nN}+i)$ by $\mathrm{y}_{\mathrm{i}}(\mathrm{n})$ from Equation (2) into Equation (4), $Y(k)$ has the form as: 


$$
\begin{aligned}
Y(k) & =\sum_{n=0}^{N-1} \sum_{i=0}^{N-1} y_{i}(n) e^{-j \frac{2 \pi}{N^{2}}(n N+i) k} \\
& =N \sum_{i=0}^{N-1} a_{i} e^{-j \frac{2 \pi}{N^{2}} k i} \underbrace{\frac{1}{N} \sum_{n=0}^{N-1} e^{-j \frac{2 \pi}{N}(k-i) n}}_{\delta\left((k-i)_{N}\right)} .
\end{aligned}
$$

Replacing $\frac{1}{N} \sum_{n=0}^{N-1} e^{-j \frac{2 \pi}{N}(k-i) n}$ by $\delta\left((k-i)_{N}\right)$ in Equation (5), we have the equation as:

Where $\delta\left((k-i)_{N}\right)=\left\{\begin{array}{ll}0, & k \neq p N+i \\ 1, & k=p N+i\end{array}\right.$ that means,

$$
Y(k)=N \sum_{i=0}^{N-1} a_{i} e^{-j \frac{2 \pi}{N^{2}} k i} \delta\left((k-i)_{N}\right)
$$

$$
\begin{aligned}
Y(p N+i) & =Y_{p}(i) \\
& =\left\{\begin{array}{ll}
0, & k \neq p N+i \\
N a_{i} e^{-j \frac{2 \pi}{N^{2}}(p N+i) i}, & k=p N+i
\end{array} \quad i, p=0,1, \cdots, N-1 .\right.
\end{aligned}
$$

Equation (7) indicates that each data symbol $a_{i}$ is modulated on $p=N$ subcarriers, and the total spectrum of the signal is spread $N$ times. Figure 4 shows the spectrum of the ISS-OFDM signal with subcarrier number $N=4$. In this case, the signal spectrum of ISS-OFDM is spread 4 times and contains 4 subbands. Each of the subbands contains the whole data information of the baseband signal.

\subsubsection{Cyclic Prefix and Pulse Shaping}

After generation of each ISS-OFDM symbol, the cyclic prefix is inserted to the ISS-OFDM symbol. The cyclic prefix is a very crucial part which is used to combat the ISI and inter channel interference (ICI) introduced by multipath fading channels through which the signal is propagated. As we discussed above, the main problem of ISI is from the energy leaking from one symbol to the other neighbor symbol. Our objective is to confine one symbol energy from leaking. Cyclic prefix insertion can effectively solve the problem.

A pulse shaping technique is employed to further reduce ISI. In the simulation of the ISS-OFDM transmitted signal, pulse shaping is implemented by using a pulse that is shaped like a Sinc function. 


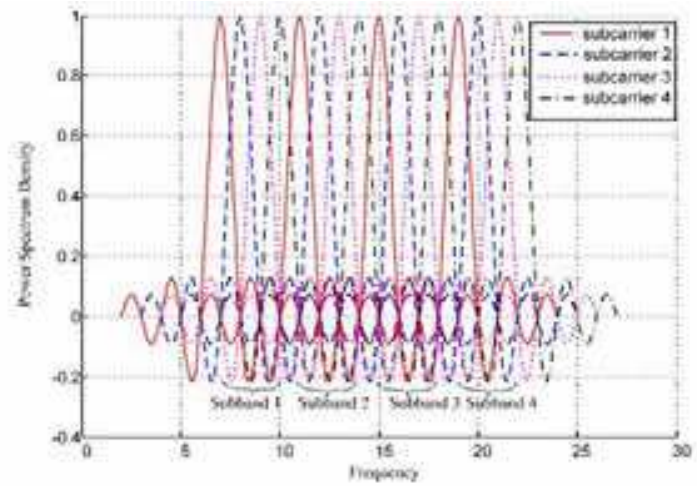

Fig. 4. Spectrum of ISS-OFDM symbol with subcarrier number $N=4$.

\subsubsection{Multiple Subband Signal Profile}

After pulse shaping, the transmitted ISS-OFDM signal has a wide bandwidth. If the bandwidth of the pulse shaping filter is designed to equal to the bandwidth of the ISS-OFDM signal frequency band, the transmitted signal has $N$ subbands and each of the subbands consists of $N$ subcarriers as displayed in Figure 5.

If the bandwidth of the filter is designed to be equivalent to $M$ out of $N(M<N, N)$ is the number of subcarriers of the base band signal) subbands of the ISS-OFDM spread spectrum signal, only $M$ subbands of the ISS-OFDM signal are transmitted, or only $M$ subbands of the $N$ subband ISS-OFDM signal are received, and the transmitted information will be recovered according to the $M$ subbands. In the extreme case, if the filter pass band is equal to one subband of the ISS-OFDM signal, the ISS-OFDM system is equivalent to the conventional OFDM system.

\subsection{Reception of Multiple Subband Signal}

Before discussing the signal reception, we take a look at the channel impacts on the transmitted signal. We may assume that the transmitted ISS-OFDM signal bandwidth BISS_OFDMis greater than the coherence bandwidth (A $f)_{c}$, BISS_OFDM (A $\left.f\right)_{c}^{\prime}$ thus the channel can be considered as a frequency-selective fading channel. The ISS-OFDM signal $y(m)$ (also represented by $Y(k)$ in the frequency domain) with spectrum spreading is

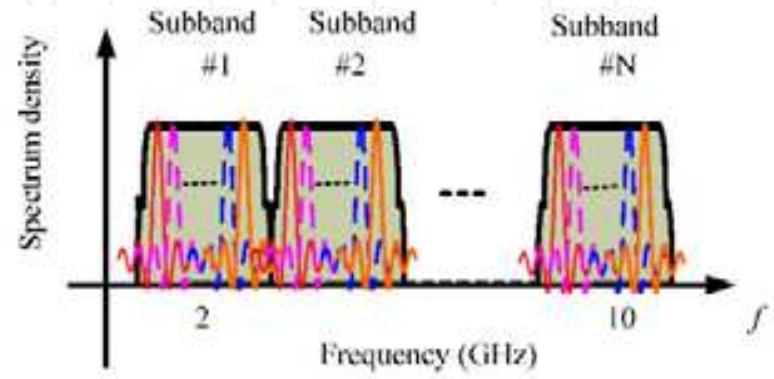

Fig. 5. Spectrum of ISS-OFDM signal. 
transmitted into the frequency selective multipath fading channel with impulse response $h(m)$ (denoted as $H(k)$ in the frequency domain). Meanwhile, the AWGN noise $\mathrm{v}(\mathrm{m})$ and (denoted as $V(k)$ in the frequency domain) and interference $j(m)$ (denoted as $J(k)$ in the frequency domain) is added to the signal. The channel model can be expressed in the form:

$$
h(m)=\sum_{i=0}^{N^{2}-1} \alpha_{i}(m) \delta\left(m-m_{i}\right),
$$

where $a_{i}(\mathrm{~m})$ is the multipath fading channel attenuation factor on the $m^{\text {th }}$ path and $m_{i}$ is the propagation delay for the $m^{\text {th }}$ path. Correspondingly, the channel frequency response is written as:

$$
H(k)=\sum_{m=0}^{N^{2}-1} h(m) e^{-j \frac{2 \pi}{N^{2}} k m} .
$$

At receiver, the received equivalent low-pass signal is viewed as the convolution between $h(m)$ and $y(m)$ plus noise and interference as follows

$$
r(m)=h(m) \otimes y(m)+v(m)+j(m)
$$

where " $\otimes$ " denotes convolution, $\mathrm{v}(\mathrm{m})$ represents the complex-valued white Gaussian noise process corrupting the signal and $j(m)$ is the interference from the coexisting other systems. The receiver structure, as displayed in Figure 1, consists of the inverse process to the transmitter. The OFDM demodulation is realized by using $N$ parallel FFTs, each of which is of size $N$. After CP removal, the serial data stream in the block of $N^{2}$ is demodulated by using N FFT operations. The output signal of the demodulator is equalized in parallel in the frequency domain, and then combined by using a maximal ratio combining (MRC) technique. The combined results are the decision variables which can be used for data detection, BER system performance computation and system performance analysis. To obtain the data vector for each FFT operation, the received time domain signal $r(m)$ is split into $N$ groups, each group is demodulated by using an FFT operation with the size $N$. After the demodulation, the channel distortion needs to be compensated using a frequency domain minimum mean square error (MMSE) equalization technique. To use MMSE, we must find out how each parallel FFT output is related to the channel coefficient and the transmitted data symbol. The first step is to derive the mathematical expressions of the received signal in the time domain at the input of the receiver in terms of the channel frequency response and the transmitted data symbol.

\subsubsection{Time domain Signal at the Receiver Input}

For deriving the time domain signal model, we firstly gain its frequency domain signal. In the frequency domain, Equation (9) is equivalent to the output of the FFT demodulator:

$$
R(k)=H(k) \cdot Y(k)+V(k)+J(k),
$$


where $\mathrm{R}(\mathrm{k}), \mathrm{Y}(\mathrm{k}), H(k), V(k), J(k)$ denote the frequency representations of the received signal $r(m)^{\prime}$ the transmitted signal $y(m)^{\prime}$ the channel impulse response $h(m)$, the noise $\mathrm{v}(\mathrm{m})$ and $\mathrm{j}(\mathrm{m})$, respectively. In the following section, the system transmitter and receiver architecture are introduced.

$$
Y(k)=Y(p N+i)= \begin{cases}0, & k \neq p N+i \\ N a_{i} e^{-j \frac{2 \pi}{N^{2}}(p N+i) i}, & k=p N+i\end{cases}
$$

Substituting $Y(k)$ from Equation (11) into $R(k)$, the received signal in the frequency domain can be rewritten as follows:

$$
R(k)=N a_{i} e^{-j \frac{2 \pi}{N^{2}} k i} H(k)+V(k)+J(k), \quad k=0,1, \cdots, N^{2}-1 .
$$

It can be observed from Equation (12) that the sample at the th position is repeated in every $N$ samples of the $N^{2}$ samples of $R(k)$. Equation (11) also indicates that the energy of each data symbol $a_{i}$ is distributed on the $(p N+i)^{\text {th }}, p=0,1,-, N-1$, subcarriers. That means that the energy of $a_{i}$ is distributed at the position $i$ of each subband with $N$

subcarriers. Each subcarrier has a phase shift, which must be compensated by the equalization in the frequency domain before the signal can be combined. The effect of the equalization and combining is to compensate for the phase shift in the channel and to weight the signal by a factor that is proportional to the signal strength.

The signal in the time domain at the input of the receiver is the $N^{2}$-point inverse discrete Foruier transform of $R(k)$, i.e.,

$$
r(m)=\operatorname{IFFT}(R(k))=\frac{1}{N^{2}} \sum_{k=0}^{N^{2}-1}(R(k)+V(k)+J(k)) e^{j \frac{2 \pi}{N^{2}} k m} .
$$

Substituting $R(k)$ from Equation (12) into Equation (13), and not considering $J(k)$ impacts, the received signal in the time domain $r(m)$ can be rewritten as:

$$
r(m)=\frac{1}{N} \sum_{k=0}^{N^{2}-1} \sum_{i=0}^{N-1} a_{i} e^{-j \frac{2 \pi}{N^{2}} k(i-m)} \delta\left((i-k)_{N}\right) H(k)+\frac{1}{N^{2}} \sum_{k=0}^{N^{2}-1} V(k) e^{j \frac{2 \pi}{N^{2}} k m} .
$$

\subsubsection{Receiver Signal Filtering}

Since the transmitted signal consists of multiple subbands, each of which has the whole transmitted information of the baseband signal, the receiver can obtain the received signal with a different number of subbands by filtering the received signal using a filter with different parameters (B. C. Jung, 2007) . By adjusting the parameters of the filter according to 
the different radio scenarios, the bandwidth of the signal used for demodulation can have different number of subbands.

Figure 6 displays an example of the signal spectrum before the receiver filter and after the receiver filter. The received signal contains $N$ subbands, where $N$ is the number of the subbands of the transmitted signal. After filtering, we can see that the received signals can be in different forms with a different number of subbands. Each subband contains the whole transmission information. If the pass band filter is configured to be equivalent to the bandwidth of 6 subbands of the transmitted signal as displayed in the green shadow, the signal used for demodulation has 6 subbands. The others will be filtered because of interference on the other subands, or because of channel bandwidth limitations. The filtered signal can be used to recover transmitted information, and receiver performance can satisfy different requirements in practical application scenarios.

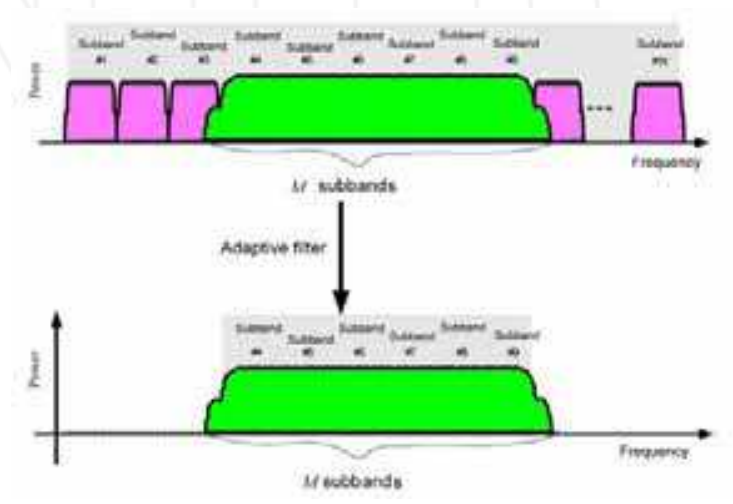

Fig. 6. Adaptive filtering at receiver.

Since only some subbands of the received signal are used for recovering the information, the process of filtering leads to a problem of signal energy loss. In consequence, it causes the reduction in power efficiency. Thus, this problem requires compromise between the selection of the subband number and signal to noise ratio. In the section on adaptive subband selection we will discuss the trade-off strategy.

\subsubsection{Deinterleaver}

Deinterleaver is to retrieve the order of the data sequence before the interleaving at the transmitter. Corresponding to the periodical interleaving algorithms used at the transmitter end, the deinterlever must be implemented by using the corresponding periodical algorithms.

\subsubsection{Demodulation Using Multiple FFTs}

After the deinterleaver, the signal in the time domain is split into $N$ data streams, each of which can be demodulated using one FFT operation. Thus, to demodulate the $N \times N$ data samples simultaneously, $N$ parallel FFTs, each in size $N$, are needed. In this way, the 
demodulation of the received signal is completed by employing $N$ parallel FFT operations. The deinterleaved signals can be written as:

$$
r_{i}(n)=r(n N+i)=r(m), \quad i=0,1, \cdots, N-1, n=0,1, \cdots, N-1 .
$$

To derive the frequency domain representation of $r_{i}(n)$, we can perform the FFT operation on $r_{i}(n)$. The output of the demodulation can be obtained as follows:

$$
R_{i}(k)=\sum_{n=0}^{N-1} r_{i}(n) e^{-j \frac{2 \pi}{N} k n}=\sum_{n=0}^{N-1} r(n N+i) e^{-j \frac{2 \pi}{N} k n}, \text { where } k=0,1, \cdots, N-1 .
$$

Substituting $r(m)$ in Equation (14) into the above equation, $R_{i}(k)$ can be written as follows:

$$
\begin{aligned}
& R_{i}(k)=\frac{1}{N} \sum_{n=0}^{N-1}\left[\sum_{k^{\prime}=0}^{N N-1} \sum_{i^{\prime}=0}^{N-1} a_{i} e^{-j \frac{2 \pi}{N N} k^{\prime}\left(i^{\prime}-n N-i\right)} \delta\left(\left(k^{\prime}-i^{\prime}\right)_{N}\right) H\left(k^{\prime}\right)+\frac{1}{N} \sum_{k^{\prime}=0}^{N N-1} V\left(k^{\prime}\right) e^{j \frac{2 \pi}{N N} k^{\prime}(n N+i)}\right] e^{-j \frac{2 \pi}{N} k n} \\
& =\sum_{k^{\prime}=0}^{N-1} \sum_{i^{\prime}=0}^{N-1} a_{i} e^{j \frac{2 \pi}{N N k^{\prime}\left(i-i^{\prime}\right)} \delta\left(\left(i^{\prime}-k^{\prime}\right)_{N}\right) H\left(k^{\prime}\right)} \underbrace{\frac{1}{N} \sum_{n=0}^{N-1} e^{j \frac{2 \pi}{N}\left(k^{\prime}-k\right) n}}_{\delta\left(\left(k^{\prime}-k\right)_{N}\right)} \\
& +\frac{1}{N N} \sum_{k^{\prime}=0}^{N N-1} V\left(k^{\prime}\right) e^{j \frac{2 \pi}{N N} k^{\prime} i} \underbrace{\frac{1}{N} \sum_{n=0}^{N-1} e^{j \frac{2 \pi}{N}\left(k^{\prime}-k\right) n}}_{\delta\left(\left(k^{\prime}-k\right)_{N}\right)} .
\end{aligned}
$$

Since $k^{\prime}-\mathrm{k}$ must be integer multiples of $N$, i.e., $\mathrm{k}^{\prime}-k=\mathrm{qN}$, we express $k^{\prime}=q N+\mathrm{k}$, and $R(k)$ is rewritten as

$$
\begin{aligned}
& R_{i}(k)=\sum_{i=0}^{N-1} a_{i} \sum_{k^{\prime}=0}^{N N-1} H\left(k^{\prime}\right) e^{j \frac{2 \pi}{N N} k^{\prime}\left(i-i^{\prime}\right)} \delta\left(\left(i^{\prime}-k^{\prime}\right)_{N}\right) \underbrace{\delta\left(\left(k^{\prime}-k\right)_{N}\right)}_{k^{\prime}=q N+k}+\frac{1}{N} \sum_{k^{\prime}=0}^{N N-1} V\left(k^{\prime}\right) \underbrace{\delta\left(\left(k^{\prime}-k\right)_{N}\right)}_{k^{\prime}=q N+k} e^{j \frac{2 \pi}{N N} k^{\prime} i} \\
& =\sum_{i^{\prime}=0}^{N-1} a_{i} \sum_{q=0}^{N-1} H(q N+k) e^{-j \frac{2 \pi}{N N}(q N+i)\left(i^{\prime}-i\right)} \underbrace{\delta\left(\left(q N+k-i^{\prime}\right)_{N}\right)}_{i^{\prime}=k}+\frac{1}{N} \sum_{q=0}^{N-1} V(q N+k) e^{j \frac{2 \pi}{N N}(q N+i) i}
\end{aligned}
$$

Again, since $q N+k$ - i' must be integer multiples of $N$, we have $i=k$ and $\mathrm{R}(\mathrm{k})$ is finally expressed as

$$
\begin{aligned}
R_{i}(k) & =a_{k} \sum_{q=0}^{N-1} H(q N+k) e^{-j \frac{2 \pi}{N N}(q N+i)(k-i)}+\frac{1}{N} \sum_{q=0}^{N-1} V(q N+k) e^{j \frac{2 \pi}{N N}(q N+k) i} \\
& =a_{k} \underbrace{\sum_{q=0}^{N-1} e^{j \frac{2 \pi}{N} q(i-k)} H(q N+k) e^{-j \frac{2 \pi}{N N} i(k-i)}}_{C_{k, i}}+\underbrace{\frac{1}{N} \sum_{q=0}^{N-1} V(q N+k) e^{j \frac{2 \pi}{N N}(q N+k) i}}_{v_{k, i}}=a_{k} C_{k, i}+v_{k, i},
\end{aligned}
$$


where

$C_{k, i}=\sum_{q=0}^{N-1} e^{j \frac{2 \pi}{N} q(i-k)} H(q N+k) e^{-j \frac{2 \pi}{N N} i(k-i)} \quad$ is an equivalent channel coefficient and

$v_{k, i}=\frac{1}{N} \sum_{q=0}^{N-1} V(q N+k) e^{j \frac{2 \pi}{N N}(q N+k) i} \quad$ is the independent additive noise.

Now the relationship among each demodulated output $R(k)$, the equivalent channel coefficient , transmitted signal $a k$, and noise $v \&_{i}$ is clearly shown in Equation (15).

\subsubsection{Channel Compensation}

Based on the analysis above, we can observe that the channel introduces distortion on the transmitted signal. The distortion can be compensated by employing frequency doamin equalization methods (W. G. Jeon, 1999) before the transmitted date bits can be recovered.

To compensate for the channel distortion, the frequency domain equalizer is used as shown in Figure 7.

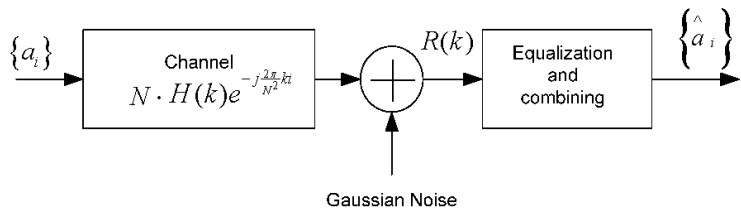

$V(k)$

Fig. 7. Frequency domain equalizer.

According to the received signal and channel models, we can derive the algorithms for computing the estimate $\left\{a_{i}\right\}$ at the output of the frequency domain equalizer.

We start the derivation process from the output of the demodulator. The demodulator output signal in the frequency domain with multipath distortions is given as in Equation (12), which is rewritten as follows:

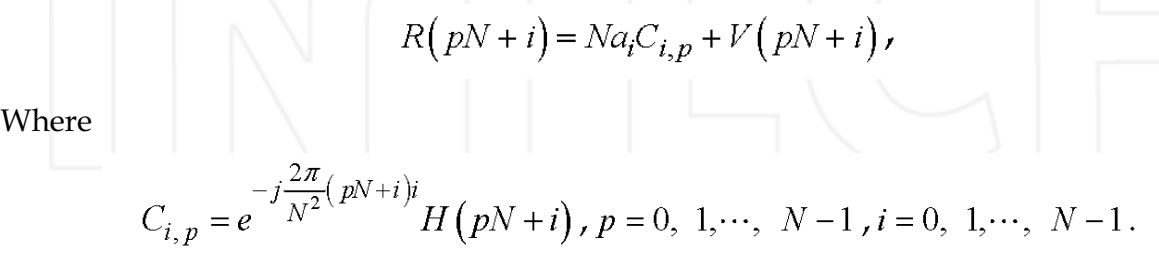

From the equation above, it can be seen that $\mathrm{R}(p N+\mathrm{i})$ is composed of $N$ groups, each group forms one subband which consists of $N$ subcarriers, and each subcarrier consists of the corresponding weighted signal and noise. Generally, we assume that the received signal contains $M$ subbands, $M<N$, and use the $M$ subbands to demodulate the transmitted signal. The structure of $\mathrm{R}(p N+\mathrm{i})$ can be illustrated in Figure 8 . 


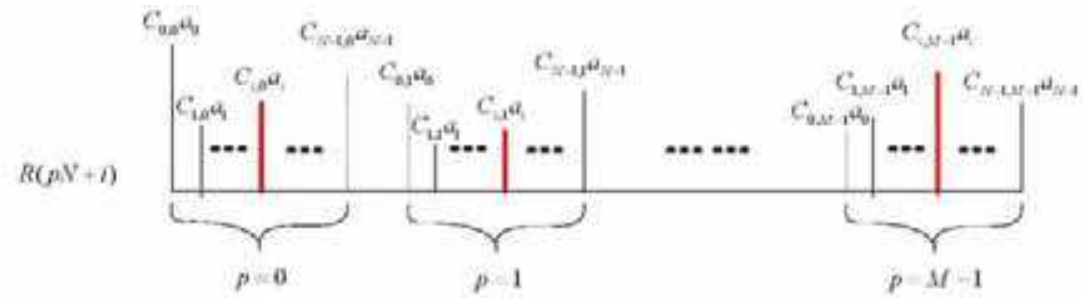

Fig. 8. The structure of $R(k)$.

The transmitted data symbol $a_{i}, i=0,1,-, N-1$, can be recovered from $\mathrm{R}(p N+\mathrm{i}), p=0,1$, ..., $M-1$, which can be expressed in matrix form as follows:

$$
\left(\begin{array}{c}
R(i) \\
R(N+i) \\
\vdots \\
R((M-1) N+i)
\end{array}\right)=N\left(\begin{array}{c}
C_{i, 0} \\
C_{i, 1} \\
\vdots \\
C_{i, M-1}
\end{array}\right) a_{i}+\left(\begin{array}{c}
V(i) \\
V(N+i) \\
\vdots \\
V((M-1) N+i)
\end{array}\right) .
$$

According to the above matrix equation, a minimum mean squared error (MMSE) estimate of $a_{i}$ can be obtained as follows:

$$
\begin{gathered}
\left.\hat{a}_{i}=\frac{1}{N}\left(\begin{array}{lll}
C_{i, 0}^{* \prime \prime} & C_{i, 1}^{*} & C_{i, M-1}^{*}
\end{array}\right)\left(\begin{array}{c}
C_{i, 0} \\
C_{i, 1} \\
\vdots \\
C_{i, M-1}
\end{array}\right)+\frac{1}{S N R}\right)^{-1} \cdot\left(\begin{array}{llll}
C_{i, 0}^{*} & C_{i, 1}^{*} & \cdots & C_{i, M-1}^{*}
\end{array}\right)\left(\begin{array}{c}
R(i) \\
R(N+i) \\
\vdots \\
R((M-1) N+i)
\end{array}\right) \\
=\frac{1}{N} \sum_{q=0}^{M-1} \frac{C_{i, q}^{*}}{\sum_{p=0}^{M-1}\left|C_{i, p}\right|^{2}+\frac{1}{S N R}} R(q N+i)^{\prime}
\end{gathered}
$$

where SNR is the signal-to-noise ratio before the MMSE equalization.

To analyze the system BER performance, the normalized MMSE can be expressed as

$$
\varepsilon_{\min }^{2}=\left(\operatorname{SNR}\left(\begin{array}{llll}
C_{i, 0}^{*} & C_{i, 1}^{*} & \cdots & C_{i, M-1}^{*}
\end{array}\right)\left(\begin{array}{c}
C_{i, 0} \\
C_{i, 1} \\
\vdots \\
C_{i, M-1}
\end{array}\right)+1\right)^{-1}=\frac{1}{S N R \sum_{p=0}^{M-1}\left|C_{i, p}\right|^{2}+1} .
$$

Therefore, the output signal-to-noise ratio after MMSE equalization is

$$
\gamma_{o u t}=\frac{1-\varepsilon_{\mathrm{min}}^{2}}{\varepsilon_{\mathrm{min}}^{2}}=\frac{1}{\varepsilon_{\mathrm{min}}^{2}}-1=S N R \sum_{p=0}^{M-1}\left|C_{i, p}\right|^{2}
$$


Assuming QPSK modulation for data symbols and making a Gaussian distribution approximation for the intersymbol interference after MMSE equalization, we finally obtain the expression for the average BER using the $Q$ function

$$
P_{e}^{(M M S E)}=E\left(Q\left(\sqrt{\gamma_{\text {out }}}\right)\right)
$$

where $E$ () denotes ensemble average over channel coefficients.

\subsubsection{Maximal Ratio Combining}

From Equation (16) we see that a data symbol $a_{i}$ is transmitted on different subcarriers and experiences independent fading. Thus, another effective way to gain frequency diversity is to directly use the maximal ration combining (MRC) technique to collect signal energy from all information bearing subcariers. In this way the system diversity can be made full use of and the best system performance can be achieved.

Similar to the above MMSE equalization, we assume that only $M$ subbands, $M<N$, are used to recover the transmitted data symbols. The MRC process proceeds as follows.

First, we multiply the conjugated channel coefficient $C_{i} \wedge$ with the received $\mathrm{R}(p N+\mathrm{i})$ to compensate the channel phase shift and weight the information bearing subcarrier with a gain proportional to the signal strength. Then, all the weighted subcarriers corresponding to the same data symbol $a_{i}$ are combined to produce the decision variable $U_{i}$ for the detection of $a_{i}$. That is,

$$
U_{i}=\sum_{p=0}^{M-1} C_{i, p}^{*} R(p N+i)
$$

To analyze the BER performance of the MRC technique, we substitute $R(p N+i)$ in the above equation with Equation (16) and have

$$
U_{i}=N \sum_{p=0}^{M-1}\left|C_{i, p}\right|^{2} a_{i}+\sum_{p=0}^{M-1} C_{i, p}^{*} V(p N+i) .
$$

The output signal-to-noise ratio after MRC can be obtained as

$$
\gamma=\frac{\left(N \sum_{p=0}^{M-1}\left|C_{i, p}\right|^{2}\right)^{2} E\left(\left|a_{i}\right|^{2}\right)}{E\left(\left|\sum_{p=0}^{M-1} C_{i, p}^{*} V(p N+i)\right|^{2}\right)}=\sum_{p=0}^{M-1}\left|C_{i, p}\right|^{2} S N R
$$


where $\quad S N R=\frac{N^{2} E\left(\left|a_{i}\right|^{2}\right)}{E\left(|V(p N+i)|^{2}\right)} \quad$ is the signal-to-noise ratio before MRC.

For QPSK modulated data symbols, the average error probability can be therefore written as:

$$
P_{e}^{(M R C)}=E(Q \sqrt{\gamma})
$$

From Equation (18) and Equation (19) we see that the MMSE equation and the MRC have the same performance for the serial demodulation.

\subsubsection{BER Performance}

For diversity performance comparison, the normalized signal-to-noise ratio $E_{b} / N o$ is more often used than $S N R$. In this section, we briefly discuss the normalization method in our system.

$E_{b} / \mathrm{No}$ is the ratio of bit energy to noise power spectral density, which is used to evaluate the BER performance. Thus, it is desirable to obtain the relation between $S N R$ and $E b / N o$, so that we can obtain the system BER performance as a function of $E_{b} / \mathrm{No}$.

In Figure 9, let er ${ }^{2}$. and $\mathrm{er}^{2}$ denote the signal variance, or average signal power, and average noise power, respectively. $N$ is the number of subcarriers used to modulate data symbols. $E_{s}$ $E b$, No denote QPKS symbol energy, bit energy and noise power spectral density, respectively. $M(M<N)$ denotes the number of subbands of the received signal after the receiver filter. $M=N$ means that all the subbands of the transmitted signal are used to recover the transmission information. Thus, the relation between $S N R$ and No can be expressed as:

$$
\begin{aligned}
S N R=\frac{\sigma_{x}^{2}}{\sigma_{v}^{2}} & =\frac{M N \sigma_{x}^{2}}{M N \sigma_{v}^{2}}=\frac{M N \sigma_{x}^{2} \cdot T \cdot M N}{M N \sigma_{v}^{2} \cdot T \cdot M N}=\frac{E_{s} \cdot N}{M N \sigma_{v}^{2} \cdot T \cdot M N} \\
& =\frac{E_{s} \cdot N}{N_{0} \cdot \frac{1}{T} \cdot T \cdot M N}=\frac{E_{s}}{N_{0} \cdot M} \cdot=\frac{2}{M} \frac{E_{b}}{N_{0}} .
\end{aligned}
$$

According to the above relationship between $S N R$ and $E b / N o$, the system BER performance expression in Equation (19) can be rewritten as: 


$$
P_{e}^{(M R C)}=E\left(Q\left(\sqrt{\sum_{p=0}^{M-1}\left|C_{i, p}\right|^{2} \frac{2}{M} \frac{E_{b}}{N_{0}}}\right)\right) .
$$

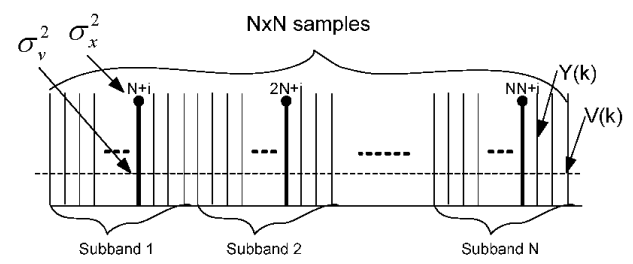

Fig. 9. SNR normalization.

\section{Adaptive Subband Selection Method}

In previous section, the multiple subband ISS-OFDM signal is presented, which consists of $N$ subbands, each containing $N$ subcarriers. When the signal passes through a multipath fading channel, the received signal experiences fading in different subbands. Meanwhile, interference signals will be imposed on the transmitted subands.

\subsection{Interference avoidance}

To avoid interference at the receiver so that system performance can be improved, the interfered subband(s) should be removed from the received signal if the interference power lever is above a predetermined threshold. The mechanism is explained in more detail below. Assume that the value of the threshold is $Y 0$ (we will discuss how to derive it in the next section). If the interference superimposed on a subband is greater than the threshold value Y0, then this interference will cause severe adverse effects on the desired signal. In order to achieve better system BER performance, the interfered subband should be excluded. If the interference is lower than the threshold value $\mathbf{Y} \mathbf{0}$, this interference can be tolerated and the interfered subbands are kept in the transmission bands. Otherwise, if we remove the interfered subbands with interference lower than the threshold, system performance will be worse than that of the system with the interfered subbands, since when we remove the interfered subbands, the signal energy is removed at the same time. Figure 10 shows the fundamental principle of interference avoidance, in which a 4 subband OFDM signal in the unlicensed $2.4 \mathrm{GHz}$ ISM frequency band is faded and interfered with, but only two of the subbands with interference over the threshold are removed.

\subsection{Determination of Interference Thresholds}

In the previous subsection, the method of avoiding interference is discussed under the assumption of a known threshold of interference. In this subsection the thresholds of interference under different channel conditions for systems coexistence are investigated. 


\subsubsection{Thresholds in Gaussian Channels}

Assume that Msubbands of the $N$ subband signal are transmitted, each having signal power $P_{S}$. If the noise power spectral density is $\mathrm{N}_{0}$ and the total interference signal power is $P_{J}$, which is distributed in $l$ subbands of the transmitted Msubband OFDM signal, then, the total signal power of the Msubbands is $M \bullet P_{S}$, and the total signal to interference noise ratio (SINR) can be expressed as:

$$
\operatorname{SINR}_{M}=\frac{M \cdot P_{S}}{N_{0} M \cdot B+P_{J}}=\frac{S N R}{1+I N R},
$$

Where $\frac{M P_{S}}{M N_{0} B}=S N R, \frac{P_{J}}{N_{0} M B}=I N R, B$, is the bandwidth of each subband.

Let $G_{p}$ denote the processing gain for each subband, the total SINR, after considering the processing gain, is

$$
\operatorname{SINR}^{(1)}=M \cdot G_{p} \cdot \operatorname{SINR}_{M}=\frac{M \cdot G_{p} \cdot S N R}{1+I N R}
$$

Similar to SINRM, if the interfered subbands are cut off, the interference energy PJis correspondingly completely removed. Thus, the total SINR, after the interfered subbands are cut off, can be expressed as:

$$
\operatorname{SINR}_{M-l}=\frac{(M-l) \cdot P_{S}}{N_{0}(M-l) \cdot B}=S N R
$$

Similar to $S I N R^{(1)}$, after the $l$ interfered subbands are removed, the total SINR after considering the processing gain is

$$
\operatorname{SINR}^{(2)}=(M-l) \cdot G_{p} \cdot \operatorname{SINR}_{M-l}=(M-l) \cdot G_{p} \cdot S N R .
$$

Since the signal energy is distributed in the whole bands, after the $l$ interfered subbands are removed, the signal energy distributed in the $l$ subbands is also removed, and the signal energy is correspondingly reduced. In a Gaussian channel, the BER performance is determined by the normalized signal-to-noise ratio. In order to have higher

SNR after the interfered subband removal, $\operatorname{SINR} R^{(2)}$ should be higher than $\operatorname{SINR}(1)$, and thus we have the following expression:

$$
\operatorname{SINR}^{(2)}>\operatorname{SINR}^{(1)} \Rightarrow I N R>\frac{l}{M-l}=\gamma_{0}
$$

It can be seen from Equation (25) that the threshold y0 of the INR is related to the number of transmitted subbands Mand the number of interfered subbands $l$ over Gaussian channels. 
Given the interfered subband number $l$, the INR threshold decreases with the increase of the number $M$ of the transmitted subbands. If the number $M$ of the transmitted subbands is given, the INR threshold increases with the increase of the interfered subband number $l$.
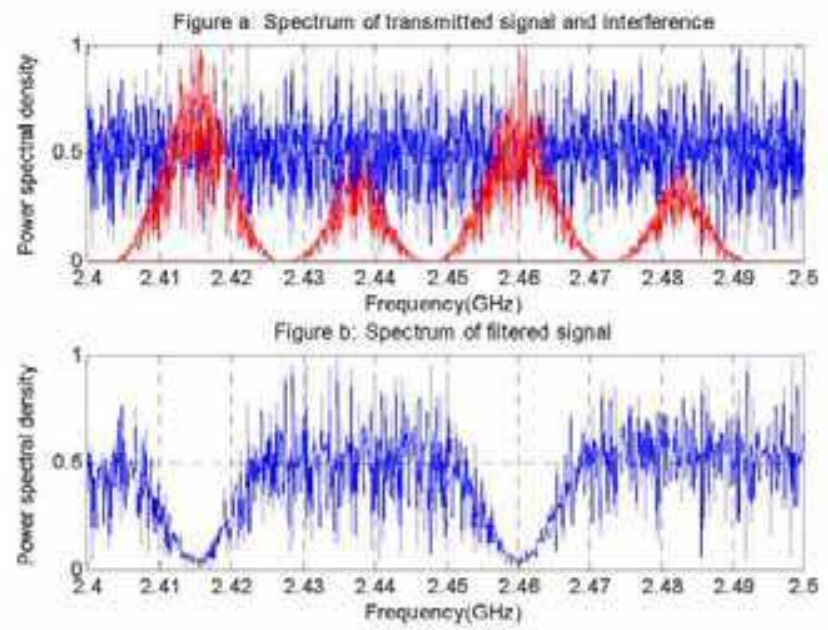

Fig. 10. Subband selection by using adaptive filter.

\subsubsection{Thresholds in Multipath Fading Channels}

In a multipath fading channel, since channel fading is random, we can not obtain the same thresholds as those in the Gaussian channel. However, it is possible for us to get an estimation of the thresholds by statistically analyzing system BER performance in a multipath fading channel.

Recalling Equation (23) and considering the multipath fading effects, system BER performance before the interfered subbands are removed can be expressed by using the $Q$ function as:

$$
P_{e}^{(1)}=E\left[Q\left(\sqrt{\sum_{p=0}^{M-1}\left|H_{p}\right|^{2} S I N R^{(1)}}\right)\right]=E\left[Q\left(\sqrt{\sum_{p=0}^{M-1}\left|H_{p}\right|^{2} \frac{S N R}{1+I N R}}\right)\right],
$$

where $E()$ denotes the statistical expectation of the conditional error probability over

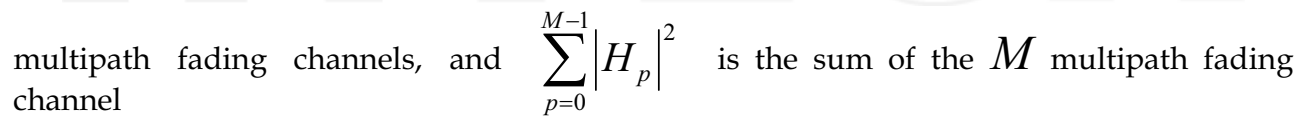


coefficients on the $M$ subcarriers. Due to the QPSK modulation, $S N R=\frac{2}{M} E_{b} / N_{0}$, which is derived in Section 2.2.7, where $E_{b} / N_{0}$ is the signal bit energy to noise spectrum density ratio. The equation above can be rewritten as:

$$
P_{e}^{(1)}=E\left[Q\left(\sqrt{\frac{1}{M} \sum_{p=0}^{M-1}\left|H_{p}\right|^{2} \frac{2 \cdot E_{b} / N_{0}}{1+I N R}}\right)\right] .
$$

Similarly, recalling Equation (24), the BER performance of the system after the interfered subbands are removed in multipath fading channels can be derived as:

$$
P_{e}^{(2)}=E\left[Q\left(\sqrt{\sum_{p=0}^{M-l-1}\left|H_{p}\right|^{2} \frac{2 \cdot E_{b} / N_{0}}{M}}\right),\right.
$$

where $E(\cdot), H_{p}, E_{b} / N_{0}$ have the same meanings as defined in Equation (24).

According to Equation (24), we simulate system BER performance with the increase of the INR when the transmission subband number is 16 and $E_{b} / N_{0}=10 d B$, which is displayed in Figure 11.

According to Equation (25) and with the same $E_{b} / N_{0}$, the BER performance of the system after the interfered subbands (its number is $l=1,2,4,8$ respectively) are removed is shown as flat lines in Figure 11. The cross points are the interference thresholds Y0 corresponding to $l=1,2,4$ and $l=8$. It can be seen from Figur 11 that the interference thresholds are $-11 \mathrm{~dB},-7.5 \mathrm{~dB},-4.0 \mathrm{~dB}$ and $1 \mathrm{~dB}$. The corresponding thresholds at the same conditions in a Gaussian channel can be calculated from Equation (25).

The interference thresholds in a multipath fading channel with transmission subbands $M=$ 2, 4, 8, 16 and 32 are presented in Table 1 . It is observed from Table 1 that the threshold discrepancies between multipath fading channel and Gaussian channel decrease with the increase of the transmission subband number and the discrepancy is approximately zero when the transmission subband number $M=32$. That is to say, we can use thresholds in a Gaussian channel to replace the thresholds in a multipath fading channel if the number of transmitted subbands is more than 32 .

\subsection{Filtering of Multiple Subband Signal}

When the multiple subband signal passes through the adaptive filter, the filtering of the multiple subband signal can be viewed as the convolution between the received multiple subband signal and the filter impulse response. 
If the bandwidth of the adaptive filter is designed to be equal to the bandwidth of the signal frequency band with $N$ subbands, after filtering, the pass-band signal has $N$ subbands, each of which has the whole transmitted information. If the bandwidth of the filter is designed to be $\mathrm{M}$ ( $M \leq N, N$ is the number of the subcarriers of the base band signal) sub-bands, only $M$ subbands of the signal are passed through the filter, or only Msubbands of the signal are received. In the case that the filter pass-band is equal to one subband of the multiple subband signal, the system model is equivalent to a conventional OFDM system.

Assume the value of the threshold is $y_{0}$. If the interference superimposed on a subband is greater than the threshold Y0, then this interference would cause severe adverse effects on the desired signal. In this case the interfered subband should be excluded. If interference is lower than the threshold Y0, the interference can be tolerated by the system and the interfered subbands should be kept in the transmission bands. These transmission signals with different numbers of subbands are very flexible to match different spectrum holes with different bandwidths.

\section{System Performance}

\subsection{PAR Simulation and Analysis}

Assume that the data symbol mapping scheme uses QPSK and the number of subcarriers $N=32$. The generated ISS-OFDM signal has $N^{2}$ samples and contains $N$ subbands, each of which contains $N$ subcarriers and carries the same data information. The transmitter is designed so that $M$ subbands are selected, $M=1, N$, respectively, and thus the transmitted signal contains $M$ subbands and the spectrum spreading factor is $M$.

We assume that the data symbol has the same energy for different spreading factors. we select the signal bandwidth to contain $M=1,2,4,8,16$ and 32 subbands respectively ( $M$ is the spreading factor), and the filtered signal is oversampled by a factor of four, which is commonly used to estimate the PAR of an analog signal from its samples. When $M=1$, there is only one signal subband to be allowed to pass through the filter, resulting in a conventional OFDM signal. When $M=2,4,8,16$ and 32, respectively, i.e., the spreading factor is 2, 4, 8, 16 and 32, the signal contains 2,4,8,16 and 32 subbands respectively.

Time domain samples of the transmitted signal $y(t)$ in the equivalent complex valued low-pass domain are approximately Gaussian distributed due to the statistical independence of carriers. Resulting PAR of $y(t)$ can be written as:

$$
\xi=\frac{\max |y(t)|^{2}}{E\left[|y(t)|^{2}\right]}
$$

where $\max |y(t)|^{2}$ is the maximum instantaneous power of the ISS-OFDM signal and $E\left[|y(t)|^{2}\right]$ is the expected value of $|y(t)|^{2}$

According to Equation (26) we can compute the PAR performance curves. Figure 12 shows the PAR performance of the ISS-OFDM transmitted signal when PAR exceeds a certain 
threshold PAR0 with the increase of the spectrum spreading factor $M$ from 1 to 32 . It can be seen from Figure 12 that with the increase of the number of subbands passing through the filter, the PAR performance is improved considerably. The most right-hand-side curve shows the PAR performance of the ISS-OFDM signal when $M$ is equal to 1 , which is the same as that of the conventional OFDM signal. As $M$ increases to 2, 4, 8, 16, and 32 respectively, the PAR gains are $0.5 \mathrm{~dB}, 1.5 \mathrm{~dB}, 3.5 \mathrm{~dB}, 5 \mathrm{~dB}$ and $7 \mathrm{~dB}$ respectively.

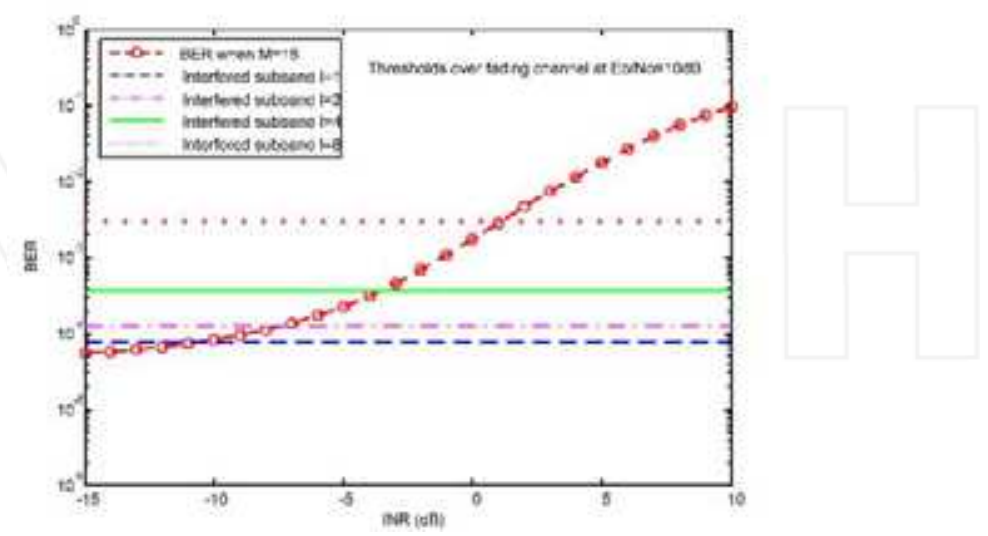

Fig. 11. INR thresholds over multipath fading channel.

\begin{tabular}{|c|c|c|c|c|c|c|c|c|c|c|}
\hline \multirow{2}{*}{$\begin{array}{l}\text { Interfered } \\
\text { Subbands }\end{array}$} & \multicolumn{2}{|c|}{$\mathrm{M}=2$} & \multicolumn{2}{|c|}{$\mathrm{M} I=4$} & \multicolumn{2}{|c|}{$M=8$} & \multicolumn{2}{|c|}{$M=16$} & \multicolumn{2}{|c|}{$\mathrm{M}=32$} \\
\hline & Gauss. & Multi. & Gauss. & Multi. & Geuss. & Multi. & Gauss. & Multi. & Gauss. & Multi. \\
\hline $1=1$ & 0 & 4.9 & -4.8 & $-1 . \bar{i}$ & -8.5 & -5.5 & -11.8 & -11.0 & -14.9 & -14.5 \\
\hline $1=2$ & - & - & 0 & 3.3 & -4.8 & -2.0 & -8.5 & -7.5 & -11.8 & -11.5 \\
\hline $1=4$ & - & - & - & - & 1) & 3.0 & -4.8 & -4.0 & -8.5 & -8.5 \\
\hline $1=8$ & - & - & - & - & - & - & 0 & 1.0 & $-4 . \overline{8}$ & -4.5 \\
\hline Discrepency & $\approx$ & & $\approx$ & & $\approx$ & & $\approx$ & & $\approx$ & .3 \\
\hline
\end{tabular}

Table 1. Threshold comparison between Gaussian and multipath channels at $E_{b} / \mathrm{N}_{0}=10 \mathrm{~dB}$.

\subsection{BER Performance}

Once the system INR thresholds are obtained, the interfered subbands with INR over the thresholds can be adaptively cut off to avoid interference. In order to verify the effectiveness of the subband adaptation in a multipath fading channel, the BER of the system without interference, with interference, and with interference removed are simulated. These are shown in Figure 13, Figure 14 and Figure 15, respectively.

Figure 13 indicates the system BER performance without interference with system transmission subband numbers $M=2,4,8,16$ and 32. It is observed that with the increase of $M$, the system BER performance is improved considerably. After $M=16$, a further increase of transmission subbands is not obviously beneficial to the improvement of the BER performance, but is helpful for interference avoidance.

Under the same conditions, the system BER with one subband interfered is displayed in Figure 14. The INR is $3 \mathrm{~dB}$. It is seen that the impact from the interference results in $5 \sim 6 \mathrm{~dB}$ degradation at the BER performance of $10^{-6}$ compared with the system performance without interference. 
As shown in Figure 15 after the interfered subband is removed when $M=4,8,16$ and 32, the system BER is improved by 0.5 $5 \mathrm{~dB}$ compared with the system performance with interference. In this case performance is very close to that without interference. However, when $M=2$ system performance becomes worse, since the subband with INR lower than the threshold ${ }^{\wedge}=4.9 \mathrm{~dB}$ is removed, which agrees with the previous analysis.

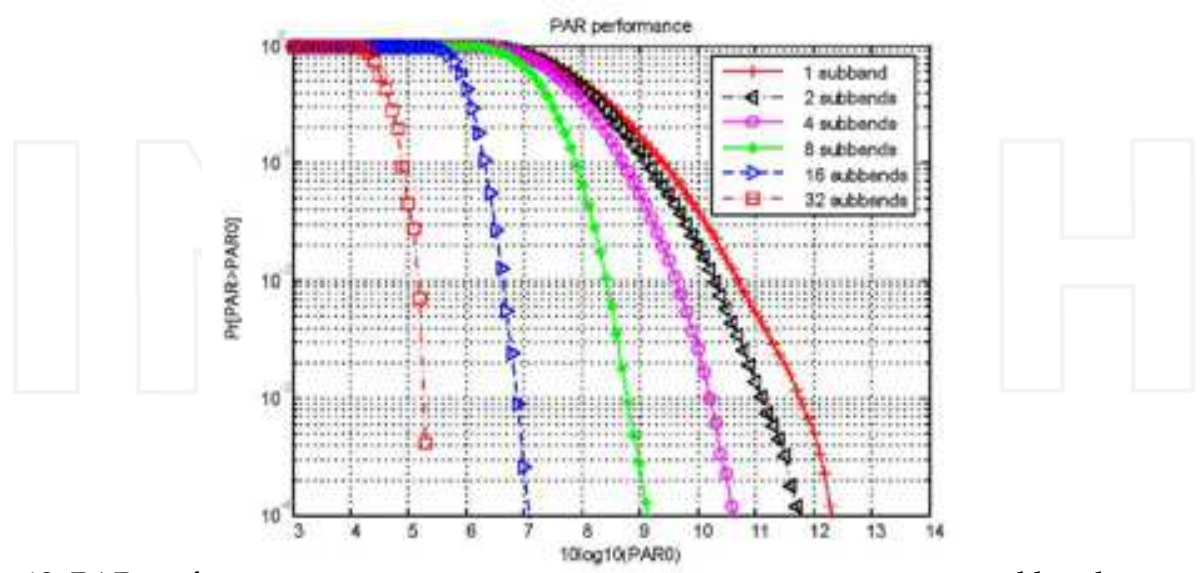

Fig. 12. PAR performance for ISS-OFDM signals with different number of subbands.

\section{Conclusions and Future Work}

In this chapter, we proposed an adaptive subband selection methods based on OFDM and cognitive radios. Since the transmission signal spectrum is spread to a wide band consisting of many subbands, and each subband contains the whole information needed to be transmitted, any one or more subband can be employed as the system transmission bandwidth to transmit information, which makes the system transmission bandwidth extremely flexible, so that the system bandwidth can be adaptively reconfigured to avoid interference and improve system performance according to the radio scene or interference conditions.

Due to the spectrum spreading of the transmitted ISS-OFDM signal, the signal transmission power is very low, which well prepares the ISS-OFDM system for use in sensor networks and wireless body area networks. It can realize this without interfering with other users.

The simulation results on system BER performance under this assumption are satisfactory and close to the theoretical analysis results. However, to some extent, we idealize the system without considering the impacts of frequency offset and synchronizations. If we add the impacts from system synchronization, system performance may degrade to some extent. Synchronization is a complex question waiting to be solved. Any fault in one of the issues of frame synchronization, symbol synchronization and subcarrier synchronization may cause the system bit error rate to be come worse. Sometimes it may result in the malfunction of the system. Therefore, ignoring system synchronization to simulate the ISS-OFDM system may not be perfect. 


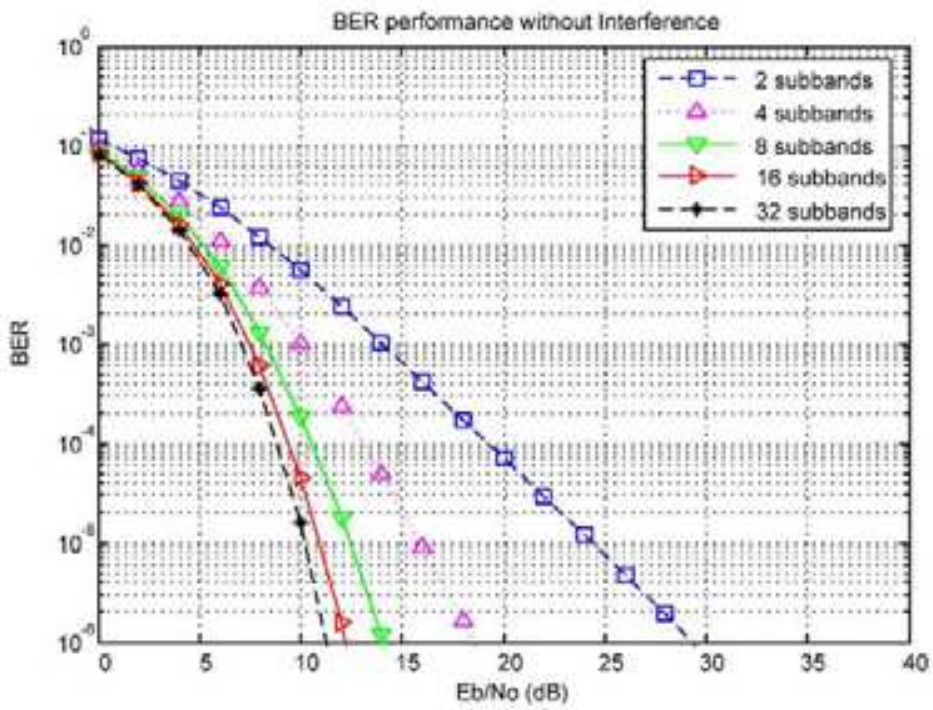

Fig. 13. BER performance without interferences in fading channel.

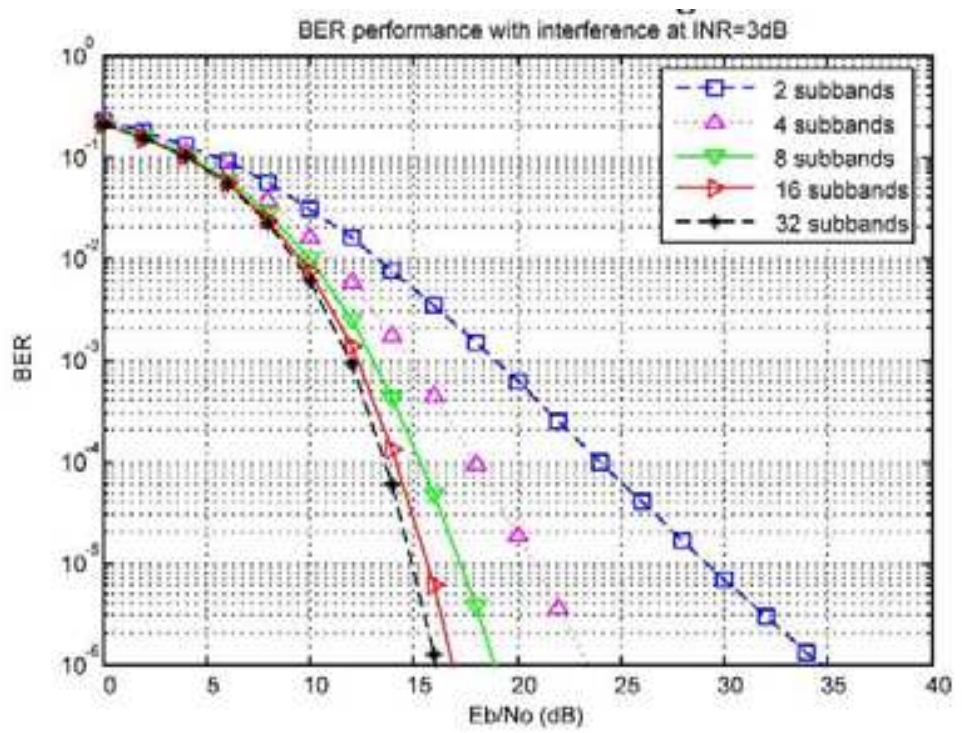

Fig. 14. BER performance with interferences in fading channel. 


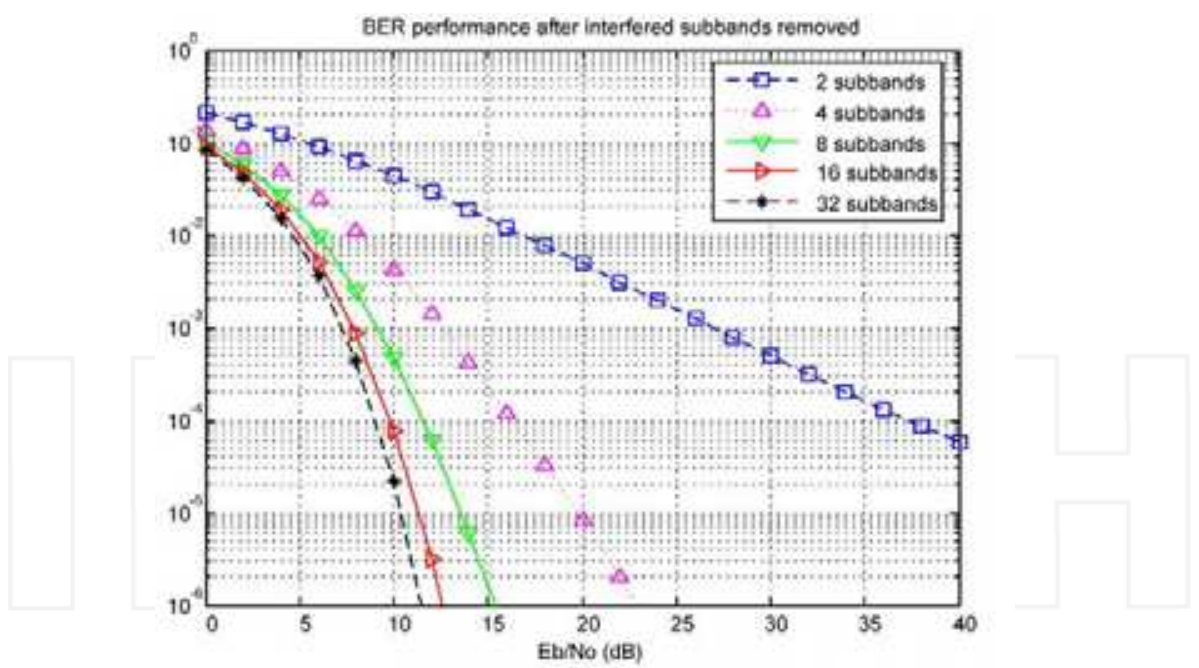

Fig. 15. BER performance after interfered subbands removed adaptively in the fading channels.

\section{References}

B. C. Jung, Y. J. Hong, D. K. Sung and S. Y. Chung, "Adaptive Sub-band Nulling for OFDM-Based Wireless Communication Systems," in IEEE Wireless Communications and Networking Conference, WCNC, pp. 1491-1495, 2007.

G. Andrea, C. Marco and D. Davide, "Coexistence Issues in Cognitive Radios Based on Ultra-Wide Bandwidth Systems," in 1st International Conference on Cognitive Radio Oriented Wireless Networks and Communications, pp. 1-5, 2006.

J. G. Proakis, Digital Communications, 4ed, New York, USA, Mc Graw Hill, 2000.

S. B. Slimane, "Peak-to-Average Power Ratio Reduction of OFDM Signals Using Broadband Pulse Shaping," in 2002 IEEE 56th Proceedings on Vehicular Technology Conference, VTC 2002-Fall, vol. 2, pp. 889-893, 2002.

S. Haykin, "Cognitive Radio: Brain-Empowered Wireless Communications," in IEEE Journal on Selected Areas in Communications, vol. 23, pp. 201-219, 2005.

T. Cooklev, Wireless Communication Standards, NewYork: Standards Information Network, IEEE Press, 2004.

W. G. Jeon, K. H. Chang and Y. S. Cho, "An Equalization Technique for Orthogonal Frequency Division Multiplexing Systems in Time-Variant Multipath Channels," in IEEE Transactions on Communications, vol. 47, pp. 27-32, 1999. 


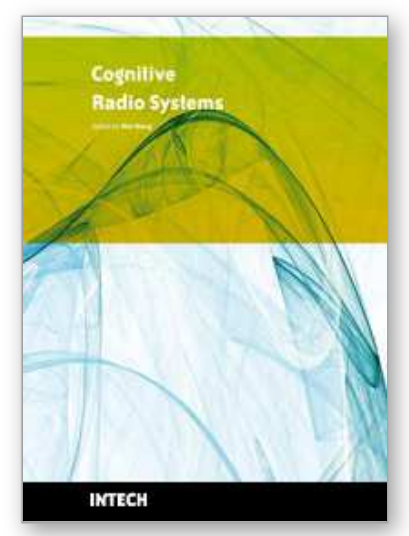

\section{Cognitive Radio Systems}

Edited by Wei Wang

ISBN 978-953-307-021-6

Hard cover, 340 pages

Publisher InTech

Published online 01, November, 2009

Published in print edition November, 2009

Cognitive radio is a hot research area for future wireless communications in the recent years. In order to increase the spectrum utilization, cognitive radio makes it possible for unlicensed users to access the spectrum unoccupied by licensed users. Cognitive radio let the equipments more intelligent to communicate with each other in a spectrum-aware manner and provide a new approach for the co-existence of multiple wireless systems. The goal of this book is to provide highlights of the current research topics in the field of cognitive radio systems. The book consists of 17 chapters, addressing various problems in cognitive radio systems.

\section{How to reference}

In order to correctly reference this scholarly work, feel free to copy and paste the following:

Pingzhou Tu, Xiaojing Huang and Eryk Dutkiewicz (2009). Adaptive Subband Selection in OFDMBasedCognitive Radios, Cognitive Radio Systems, Wei Wang (Ed.), ISBN: 978-953-307-021-6, InTech, Available from: http://www.intechopen.com/books/cognitive-radio-systems/adaptive-subband-selection-inofdm-basedcognitive-radios

\section{INTECH}

open science | open minds

\section{InTech Europe}

University Campus STeP Ri

Slavka Krautzeka 83/A

51000 Rijeka, Croatia

Phone: +385 (51) 770447

Fax: +385 (51) 686166

www.intechopen.com

\section{InTech China}

Unit 405, Office Block, Hotel Equatorial Shanghai

No.65, Yan An Road (West), Shanghai, 200040, China

中国上海市延安西路65号上海国际贵都大饭店办公楼 405 单元

Phone: +86-21-62489820

Fax: $+86-21-62489821$ 